
\title{
Phtytogeographical Distribution of Indian Vitaceae: A Report
}

\author{
Nasheez Fatma \\ Post Graduate Department of Botany, T.M. Bhagalpur University, Bhagalpur, Bihar
}

\begin{abstract}
Vitaceae comprises 14 genera and 978 species distributed throughout the world. In India 10 genera and 82 species of the family is distributed mostly in the tropical, subtropical and evergreen forests. Species like Cissus subramanyanii Shetty and Singh and Tetrastigma andamanicum (King) Suess. ex Suess. are endemic to Tamil Nadu and Andaman and Nicobar islands respectively. The Leeaceae, earlier excluded from the family, is monogeneric with about 34 species of which c. 11 occurs in India.
\end{abstract}

Keywords: Vitaceae, Leea, species, monogeneric, forest

\section{Introduction}

Vitaceae comprises 14 genera and 978 species distributed throughout the world basically in Northern Hemisphere in the tropical regions (Soejima and Wen, 2006; Xang, et al., 1998; Wen, 1999; Peros et al., 2011; Nie et al., 2010). Genus Vitis, Ampelopsis and Parthenocissus are mostly restricted to the mountainous regions of the temperate zone. Most species of Vitaceae are forest plants while some are Savanna dwellers. The Leeaceae, earlier excluded from the family Vitaceae, is monogeneric (APG III, 2009) with about 34 species of which c. 11 occur in India (Naithani, 2000). It primarily confines in Malaysia, Indochina extending to Micronesia, Melanesia i.e., tropical and subtropical Asia, Australia and Tropical Africa.

Paleontological reports suggest that earliest seeds of Vitaceae are from the late Paleocene (Chen and Manchester, 2007). Among the seed fossils, the most common species and the highest number are represented by the genus Vitis. Species of Vitis are now commonly distributed in North America and East to South Asia, forming a disjunct distribution between these two continents (Chen and Manchester, 2007). There is no wild record from Europe except for the cultivated species, Vitis vinifera (Punt et al., 2003) which suggest that the final disappearance of Vitis from Europe may have resulted from the successive climatic cooling trend during the late Cenozoic (Manchester, 1994, Wen, 1999). Despite the existence of a variety of patterns, it has been suggested that many plant taxa initially diversified in Asia and then dispersed to North America through the Bering Land Bridge (Donoghue et al., 2001). Dispersal within continents appears to have been asymmetrical, mostly westward in Eurasia but eastward in North America (Xiang \& Soltis, 2001).

\section{Material and method}

The present work deals with the phyto-geographical distribution of the family Vitaceae. The species were collected from different localities of India. The major objective proposed for the present research was to document the intensive details of the collected species and to proceed the phylogenetic study. The plants were collected either in flowering or fruiting or both and the collected specimens were processed following standard herbarium techniques (Jain and Rao, 1977) and specimens have been deposited at Bhagalpur University Herbarium (BHAG).

\section{Results and Discussion}

In India 10 genera and 82 species of the family is distributed mostly in the tropical, subtropical and evergreen forests of India (Shetty and Singh, 2000). Species like Cissus subramanyanii Shetty and Singh and Tetrastigma andamanicum (King) Suess. ex Suess. are endemic to Tamil Nadu and Andaman and Nicobar islands respectively. Many species of Ampelocissus, Nothocissus, Cissus and Pterisanthes prefer lowland tropical forests. Tetrastigma exists in lowland forests. Species like Leea alata prefer dry wood lands. Others like Leea indica, L. guineensis, L. aequata, $L$. angulata, $L$. rubra etc. have a wide range of distribution (Wen, 2007a; Nie et al., 2010). Yua occurs in China, India and Nepal (Li, 1990). The detailed phytogeographical distribution of the Indian Vitaceous species is shown in Table 1(after Shetty \& Singh 2000). 


\section{International Journal of Science and Research (IJSR) \\ ISSN (Online): 2319-7064 \\ Index Copernicus Value (2013): 6.14 | Impact Factor (2014): 5.611}

Table 1: Phyto- geographical distribution of the Indian Species of Vitaceae

\begin{tabular}{|c|c|c|c|}
\hline S.N & Species & Distribution in India & Distribution in World \\
\hline 01. & $\begin{array}{l}\text { Ampelocissus araneosa } \\
\text { (Dalz.) Planch. ex Gam. }\end{array}$ & $\begin{array}{l}\text { Evergreen forests of Western Ghats, Maharashtra, } \\
\text { Karnataka and Tamil Nadu. }\end{array}$ & ---- \\
\hline f & $\begin{array}{l}\text { Ampelocissus barbata } \\
\text { (Wallich) Planch. }\end{array}$ & $\begin{array}{l}\text { Tropical and subtropical forests, Sikkim, Uttar Pradesh } \\
\text { (Kumaon), West Bengal Arunachal Pradesh, Nagaland, } \\
\text { Mizoram, Tripura, Meghalaya, Andaman and Nicobar. }\end{array}$ & $\begin{array}{c}\text { Bhutan, Bangladesh, Myanmar, Thailand, } \\
\text { Laos and Vietnam }\end{array}$ \\
\hline 03 & $\begin{array}{l}\text { Ampelocissus } \\
\text { divaricata (Wall. ex } \\
\text { Law.) Planch. }\end{array}$ & $\begin{array}{c}\text { Evergreen forests, Uttar Pradesh Arunachal Pradesh, } \\
\text { Himachal Pradesh, Bihar, Odisha, Madhya Pradesh, } \\
\text { Andhra Pradesh and Tamil Nadu }\end{array}$ & Bhutan and Indo-China \\
\hline 04. & $\begin{array}{c}\text { Ampelocissus } \\
\text { helferi (Law.) Planch. }\end{array}$ & Andaman Islands & Myanmar \\
\hline 05. & $\begin{array}{l}\text { Ampelocissus indica } \\
\text { (L.) Planch. }\end{array}$ & $\begin{array}{c}\text { Evergreen forests of Western Ghats from Konkan } \\
\text { Southwards, Karnataka, Kerala, Goa and Tamil Nadu. }\end{array}$ & Sri Lanka \\
\hline 06. & $\begin{array}{l}\text { Ampelocissus latifolia } \\
\text { (Roxb.) Planch. }\end{array}$ & $\begin{array}{l}\text { Almost throughout India in evergreen and deciduous } \\
\text { forests. }\end{array}$ & Pakistan, Nepal and Bangladesh. \\
\hline 07. & $\begin{array}{l}\text { Ampelocissus rugosa } \\
\text { (Wall.) Planch. }\end{array}$ & $\begin{array}{c}\text { Himalaya from Gharwal eastwards and Khasia hills } \\
\text { between 1200-2500m, Uttar Pradesh, Sikkim, Arunachal } \\
\text { Pradesh and Meghalaya }\end{array}$ & Nepal and Myanmar \\
\hline 08. & $\begin{array}{l}\text { Ampelocissus } \\
\text { sikkimensis (Law.) } \\
\text { Planch. }\end{array}$ & $\begin{array}{c}\text { Subtropical forests of eastern part between } 600-1500 \mathrm{~m} \text {, } \\
\text { Assam, West Bengal, Sikkim, Arunachal Pradesh, Mizoram } \\
\text { and Meghalaya. }\end{array}$ & Nepal \\
\hline 09. & $\begin{array}{l}\text { Ampelocissus } \\
\text { tomentosa (Roth.) } \\
\text { Planch. }\end{array}$ & $\begin{array}{c}\text { Dry deciduous forests of Central and eastern India and hills } \\
\text { of } \\
\text { peninsular India upto1500m Uttar Pradesh, Andhra } \\
\text { Pradesh, Bihar, Odisha, West Bengal, Madhya Pradesh, } \\
\text { Gujarat, Maharashtra, Karnataka, Kerala and Tamil Nadu. }\end{array}$ & Bangladesh and Myanmar \\
\hline 10. & $\begin{array}{c}\text { Ampelocissus } \\
\text { whightiana Shetty } \\
\text { and Singh } \\
\end{array}$ & $\begin{array}{c}\text { Evergreen forests of Courtallum, } \\
\text { Kannikati and Muthkar vayal up } \\
\text { to } 1000 \mathrm{~m} \text { Tamil Nadu (rare). }\end{array}$ & Sri Lanka \\
\hline 11. & $\begin{array}{l}\text { Ampelopsis glandulosa } \\
\text { (Wall.) Momuj. }\end{array}$ & $\begin{array}{c}\text { Subtropical forests of Northeast India between } 600-1700 \mathrm{~m} \text {, } \\
\text { Sikkim, Manipur and Meghalaya rare). }\end{array}$ & Nepal and Myanmar \\
\hline 12. & $\begin{array}{l}\text { Ampelopsis rubifolia } \\
\text { (Wallich.) Planch. }\end{array}$ & Assam, Mizoram and Meghalaya. & Bangladesh (Sylhet). \\
\hline 13. & $\begin{array}{l}\text { Ampelopsis japonica } \\
\text { (Thunb.) Makino }\end{array}$ & $\begin{array}{c}\text { West Bengal, Sikkim, Assam, Meghalaya, Mizoram, } \\
\text { Andaman Islands. }\end{array}$ & Myanmar, China, Japan, Korea \\
\hline 14. & $\begin{array}{l}\text { Ampelopsis vitifolia } \\
\text { (Boiss.) Planch. }\end{array}$ & Jammu and Kashmir, Himachal Pradesh. & Pakistan, Iran and Afghanistan \\
\hline 15. & $\begin{array}{l}\text { Cayratia anemonifolia } \\
\text { (Zipp. ex Miq.)Suess. }\end{array}$ & Evergreen forests between $500-1800 \mathrm{~m}$ and Tamil Nadu. & Indonesia (Timor) \\
\hline 16. & $\begin{array}{l}\text { Cayratia geniculata } \\
\text { (Blume) Gag. }\end{array}$ & $\begin{array}{l}\text { Tropical and subtropical forests between 300-1200m, } \\
\text { Sikkim, West Bengal (Darjeeling dist.), Assam, Arunachal } \\
\text { Pradesh and Manipur. }\end{array}$ & $\begin{array}{l}\text { Bhutan, Laos, Indonesia (Java, Borneo) and } \\
\text { Philippines }\end{array}$ \\
\hline 17. & $\begin{array}{l}\text { Cayratia japonica } \\
\text { (Thumb.) Gag. }\end{array}$ & $\begin{array}{l}\text { Tropical and subtropical forests upto1500m West Bengal, } \\
\text { Goa, Sikkim, Arunachal Pradesh, Kerala, Assam, } \\
\text { Nagaland, Manipur, Tripura, Mizoram, Karnataka, Tamil } \\
\text { Nadu, Meghalaya, Andaman and Nicobar. }\end{array}$ & $\begin{array}{l}\text { Nepal, Myanmar, Bhutan, Malaysia, } \\
\text { Bangladesh, Japan, Vietnam, Korea, } \\
\text { Cambodia, Taiwan, and Australia } \\
\text { (Queensland). }\end{array}$ \\
\hline & $\begin{array}{l}\text { Cayratia mollissima } \\
\text { (Wall.) Gag. }\end{array}$ & $\begin{array}{c}\text { Evergreen forests of Western Ghats upto } 1000 \mathrm{~m} \text {, } \\
\text { Karnataka, Kerala, Tamil Nadu and Arunachal Pradesh. }\end{array}$ & $\begin{array}{l}\text { Myanmar, Vietnam Thailand, Cambodia, } \\
\text { and Malaysia. }\end{array}$ \\
\hline aa & $\begin{array}{l}\text { Cayratia pedata var. } \\
\text { pedata (Lam.) Gag }\end{array}$ & $\begin{array}{c}\text { Bihar, Odisha, West Bengal, Assam, Arunachal Pradesh, } \\
\text { Andhra Pradesh, Kerala, } \\
\text { Karnataka, Tamil Nadu Andaman and Nicobar }\end{array}$ & $\begin{array}{l}\text { Bangladesh, Myanmar, Thailand, Vietnam, } \\
\text { Cambodia, Indonesia (Java), Sri Lanka and } \\
\text { Philippines }\end{array}$ \\
\hline & $\begin{array}{l}\text { Cayratia pedata var. } \\
\text { glabra Gambl. }\end{array}$ & $\begin{array}{l}\text { In evergreen forests at an altitude of about 1800m, Tamil } \\
\text { Nadu (endemic). }\end{array}$ & ----- \\
\hline 20. & $\begin{array}{l}\text { Cayratia roxburghii } \\
\text { Gag. }\end{array}$ & Bihar, Kerala and Tamil Nadu & Sri Lanka \\
\hline 21. & $\begin{array}{l}\text { Cayratia tenuifolia } \\
\text { (Wight \& Arn.) Gag. }\end{array}$ & Karnataka, Kerala and Tamil Nadu & Sri Lanka and Indo-China \\
\hline 22. & $\begin{array}{l}\text { Cayratia trifolia (L.) } \\
\text { Domin }\end{array}$ & Almost throughout India at lower elevations in the hills. & $\begin{array}{c}\text { Pakistan, Nepal, China, Bangladesh, } \\
\text { Myanmar, Indo-China, Malaysia and } \\
\text { Australia. }\end{array}$ \\
\hline 23. & Cissus adnata Roxb. & $\begin{array}{c}\text { West Bengal, Assam, Sikkim, Arunachal Pradesh, Manipur, } \\
\text { Mizoram, Tripura, Meghalaya } \\
\text { and Odisha }\end{array}$ & $\begin{array}{c}\text { Sri Lanka, Nepal, Myanmar, Indonesia, } \\
\text { Bangladesh, Indo-China, Maleysia, } \\
\text { Phillipines and Australia. }\end{array}$ \\
\hline 24. & Cissus aristata Blume & $\begin{array}{c}\text { West Bengal, Assam, Sikkim, Nagaland, Mizoram, Kerala, } \\
\text { Maharashtra, Karnataka and Tamil Nadu }\end{array}$ & $\begin{array}{c}\text { Bangladesh, Maleysia, Myanmar, Sri Lanka, } \\
\text { Indo-China, Indonesia, New-Guinea and } \\
\text { Philippines. }\end{array}$ \\
\hline 25. & $\begin{array}{l}\text { Cissus arnottiana } \\
\text { Shetty and Singh }\end{array}$ & $\begin{array}{c}\text { Andhra Pradesh, Gujarat, Kerala, Maharashtra, Karnataka } \\
\text { and Tamil Nadu }\end{array}$ & Sri Lanka. \\
\hline
\end{tabular}




\section{International Journal of Science and Research (IJSR) \\ ISSN (Online): 2319-7064}

Index Copernicus Value (2013): 6.14 | Impact Factor (2014): 5.611

\begin{tabular}{|c|c|c|c|}
\hline 26. & $\begin{array}{l}\text { Cissus assamica } \\
\text { (Lawson) Craib }\end{array}$ & $\begin{array}{c}\text { Odisha, West Bengal, Assam, Arunachal Pradesh, Sikkim, } \\
\text { Manipur, Tripura and Meghalaya. }\end{array}$ & $\begin{array}{l}\text { Nepal, Bangladesh, Myanmar, Indo-China, } \\
\text { Maleysia, Bhutan and Philippines. }\end{array}$ \\
\hline 27. & Cissus discolor Blume. & $\begin{array}{c}\text { Assam, Sikkim, Arunachal Pradesh, Manipur, Meghalaya, } \\
\text { Mizoram, Maharashtra, Kerala, Karnataka, Tamil Nadu, } \\
\text { Andaman and Nicobar. }\end{array}$ & $\begin{array}{l}\text { Nepal, Bangladesh, China, Indo-China, } \\
\text { Malaysia, Indonesia (Java), Myanmar, and } \\
\text { Philippines. }\end{array}$ \\
\hline \multirow[t]{2}{*}{28.} & $\begin{array}{c}\text { Cissus elongata subsp. } \\
\text { elongate Roxb. }\end{array}$ & Assam, Sikkim, Meghalaya, Andaman and Nicobar. & Bhutan and Bangladesh \\
\hline & $\begin{array}{l}\text { Cissus elongata subsp. } \\
\text { littoralis (Talbot) } \\
\text { Shetty }\end{array}$ & Maharashtra, Goa and Karnataka. & 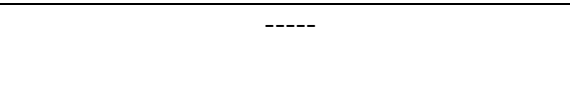 \\
\hline 29. & $\begin{array}{l}\text { Cissus glyptocarpa } \\
\text { Thw. }\end{array}$ & $\begin{array}{l}\text { Western Ghats between 300-1100m, Karnataka, Tamil } \\
\text { Nadu and Kerala. }\end{array}$ & Sri Lanka \\
\hline 30. & Cissus heyeana Planch. & Tamil Nadu and Kerala. & Sri Lanka \\
\hline 31. & Cissus latifolia Lam. & Goa, Karnataka, Tamil Nadu and Kerala. & Sri Lanka. \\
\hline 32. & Cissus nodusa Blume. & Andaman Islands. & Malaysia. \\
\hline 33. & $\begin{array}{l}\text { Cissus quadrangularis } \\
\text { L. }\end{array}$ & $\begin{array}{c}\text { Bihar, Odessa, West Bengal, Assam, Maharashtra, Goa } \\
\text { Andhra Pradesh, Kerala, Karnataka, Tamil Nadu, } \\
\text { Daman and Lakshadweep islands. }\end{array}$ & $\begin{array}{l}\text { Africa, Sri Lanka, Bangladesh, Indo-China, } \\
\text { Indonesia (Java) Myanmar, and Philippines. }\end{array}$ \\
\hline 34. & $\begin{array}{c}\text { Cissus hamaderohensis } \\
\text { Radcl.-Smith. }\end{array}$ & Mostly cultivated as an ornamental plant. & Yemen. \\
\hline 35. & $\begin{array}{l}\text { Cissus rotundifolia } \\
\text { Vahl. }\end{array}$ & Almost throughout India as ornamental plant. & $\begin{array}{l}\text { Grenoble, Arizona Florida, California, } \\
\text { Minnesota, Arab, East Africa, Gorge and } \\
\text { Zimbabwe. }\end{array}$ \\
\hline 36. & Cissus repanda Vahl. & $\begin{array}{l}\text { Bihar, Uttar Pradesh, Madhya Pradesh, Odessa, West } \\
\text { Bengal, Assam, Gujarat, Rajasthan, Goa, Andhra Pradesh, } \\
\text { Karnataka, Kerala, Maharashtra, Tamil Nadu, Tripura and } \\
\text { Arunachal Pradesh. }\end{array}$ & $\begin{array}{l}\text { Sri Lanka, Nepal, Bhutan, Bangladesh, } \\
\text { Myanmar and Indo-China. }\end{array}$ \\
\hline 37. & Cissus repens Lam. & $\begin{array}{l}\text { West Bengal, Sikkim, Arunachal Pradesh, Assam, } \\
\text { Meghalaya, Odisha, Goa, Andhra Pradesh, Karnataka, } \\
\text { Tamil Nadu, Kerala, and Andaman and Nicobar Islands. }\end{array}$ & $\begin{array}{l}\text { Nepal to Taiwan, Myanmar, Thailand, Indo- } \\
\text { China, Malaysia and Indonesia (Java). }\end{array}$ \\
\hline 38. & $\begin{array}{l}\text { Cissus spectabilis } \\
\text { (Kurz.) Planch. }\end{array}$ & West Bengal, Sikkim (endemic). & ----- \\
\hline 39. & Cissus hastata Miq. & $\begin{array}{c}\text { West Bengal, Sikkim, Arunachal Pradesh, Assam, } \\
\text { Meghalaya. }\end{array}$ & $\begin{array}{c}\text { Indo-China,Thailand, South-east Asia, } \\
\text { Australia, Malaysia, Thailand and Vietnam. }\end{array}$ \\
\hline 40. & $\begin{array}{l}\text { Cissus subramanyamii } \\
\text { Shetty and Singh }\end{array}$ & Tamil Nadu (endemic). & $\begin{array}{ll}---- \\
\end{array}$ \\
\hline 41. & Cissus trilobata Lam. & Kerala, Tamil Nadu and Lakshadweep islands. & Sri Lanka. \\
\hline 42. & Cissus vitignea L. & $\begin{array}{l}\text { Odisha, Maharashtra, Andhra Pradesh, Kerala, Karnataka } \\
\text { and Tamil Nadu. }\end{array}$ & Sri Lanka \\
\hline 3. & $\begin{array}{c}\text { issus woodrowii (Stapf. } \\
\text { ex Cooke) Santapau. }\end{array}$ & Maharashtra & $\begin{array}{ll}---- \\
--\end{array}$ \\
\hline 44. & \begin{tabular}{|c|} 
Cyphostemma \\
auriculatum (Roxb.) \\
Shetty and Singh. \\
\end{tabular} & $\begin{array}{l}\text { Bihar, Uttar Pradesh, Madhya Pradesh, Odessa, West } \\
\text { Bengal, Assam, Gujarat, Rajasthan, Maharashtra, Goa, } \\
\text { Andhra Pradesh, Kerala and Tamil Nadu. }\end{array}$ & Bangladesh and Myanmar. \\
\hline 45. & $\begin{array}{l}\text { Cyphostemma setossum } \\
\text { (Roxb.) Alston }\end{array}$ & $\begin{array}{c}\text { Dry localities in Peninsular India on dry stony hills upto } \\
\text { 500m sometimes on black soil plains, Andhra Pradesh, } \\
\text { Maharashtra, Karnataka and Tamil Nadu. }\end{array}$ & Sri Lanka \\
\hline 46. & \begin{tabular}{|c|} 
Parthenocissus \\
semicordata var. \\
semicordata (Wall.) C \\
\end{tabular} & $\begin{array}{c}\text { Himachal Pradesh, Uttar Pradesh, West Bengal, Sikkim, } \\
\text { Meghalaya and Nagaland }\end{array}$ & Pakistan, Nepal and Bhutan. \\
\hline & $\begin{array}{l}\text { var. roylei (King) } \\
\text { Raiz.\& Sax. }\end{array}$ & $\begin{array}{c}\text { Himachal Pradesh, Punjab, Uttar Pradesh, Meghalaya, } \\
\text { Kerala, and Tamil Nadu. }\end{array}$ & $\begin{array}{l}\text { Pakistan, Nepal, Bhutan, West China, } \\
\text { Myanmar, Thailand and Malaysia }\end{array}$ \\
\hline 47. & $\begin{array}{c}\text { Parthenocissus } \\
\text { thomsonii (Lawson) } \\
\text { Planch. }\end{array}$ & Meghalaya and Arunachal Pradesh. & Nepal and West and Central China. \\
\hline 48. & $\begin{array}{l}\text { Parthenocissus } \\
\text { quinquefolia (L.) } \\
\text { Planch. } \\
\end{array}$ & Cult. Sp. often in Gardens & Native of North America. \\
\hline 49. & $\begin{array}{c}\text { Parthenocissus } \\
\text { tricuspidata (Sieb. \& } \\
\text { Zucc.) Planch. }\end{array}$ & Cult. Sp. often in Gardens & Native of Japan and Central China. \\
\hline 50. & $\begin{array}{c}\text { Tetrastigma affine } \\
\text { (Gag. ex Osm) Raiz.\& } \\
\text { Sax. }\end{array}$ & $\begin{array}{c}\text { Uttar Pradesh, Assam, Arunachal Pradesh, Sikkim, } \\
\text { Meghalaya, Andaman and Nicobar }\end{array}$ & Nepal and Bangldesh. \\
\hline 51. & $\begin{array}{c}\text { Tetrastigma } \\
\text { andamanicum } \\
\text { (King.) Suess ex } \\
\text { Suess } \\
\end{array}$ & Andaman and Nicobar (endemic) & ----- \\
\hline
\end{tabular}

Volume 5 Issue 3, March 2016 


\section{International Journal of Science and Research (IJSR) \\ ISSN (Online): 2319-7064}

Index Copernicus Value (2013): 6.14 | Impact Factor (2014): 5.611

\begin{tabular}{|c|c|c|c|}
\hline 2. & $\begin{array}{l}\text { Tetrastigma } \\
\text { angustifolium (Roxb.) } \\
\text { Roxb. }\end{array}$ & West Bengal, Assam, Meghalaya and Arunachal Pradesh. & $\begin{array}{c}\text { Bangladesh, Indonesia (Sumatra) and China } \\
\text { (Hongkong). }\end{array}$ \\
\hline 53. & \begin{tabular}{|c|} 
Tetrastigma \\
bracteolatum (Wallich.) \\
Planch.
\end{tabular} & $\begin{array}{c}\text { Bihar, Assam, Sikkim, Meghalaya, Manipur, West Bengal, } \\
\text { Arunachal Pradesh }\end{array}$ & Nepal, Bhutan, Bangladesh. \\
\hline 54. & \begin{tabular}{|c|} 
Tetrastigma \\
$\begin{array}{c}\text { campylocarpum (Kurz.) } \\
\text { Planch. }\end{array}$ \\
\end{tabular} & $\begin{array}{l}\text { Uttar Pradesh, Bihar, Assam, Sikkim, West Bengal, } \\
\text { Mizoram Andaman and Nicobar. }\end{array}$ & Bangladesh, Myanmar and China (Yunnan). \\
\hline 55. & $\begin{array}{l}\text { Tetrastigma dubuim } \\
\text { (Law.) Planch. }\end{array}$ & \begin{tabular}{|c|} 
Assam, Sikkim, Mizoram, Manipur, Tripura, West Bengal, \\
Arunachal Pradesh and Meghalaya
\end{tabular} & Nepal, Bangladesh, Myanmar and China. \\
\hline 56. & $\begin{array}{l}\text { Tetrastigma gamblei } \\
\text { Shetty and Singh }\end{array}$ & Maharashtra, Karnataka and Kerala & ------ \\
\hline 57. & $\begin{array}{l}\text { Tetrastigma hookeri } \\
\text { (Lawson) Planch. }\end{array}$ & Assam and Sikkim & $\begin{array}{l}\text { Nepal, Bhutan, Bangladesh, Malaysia and } \\
\text { Indonesia (Java). }\end{array}$ \\
\hline 8. & \begin{tabular}{|c|} 
Tetrastigma \\
leucotaphyllum (Denn.) \\
Als. ex Mab.
\end{tabular} & $\begin{array}{c}\text { Assam, Meghalaya, Karnataka Tamil Nadu Odessa, } \\
\text { Andhra Pradesh and Kerala }\end{array}$ & Nepal, Bhutan and Bangladesh \\
\hline 59. & $\begin{array}{l}\text { Tetrastigma } \\
\text { nilagiricum (Miq.) } \\
\text { Shetty }\end{array}$ & Karnataka Tamil Nadu and Kerala & Sri Lanka \\
\hline 60. & $\begin{array}{l}\text { Tetrastigma obovatum } \\
\text { Gag. }\end{array}$ & Sikkim, Arunachal Pradesh, Nagaland and Meghalaya & Bhutan, Bangladesh and Myanmar. \\
\hline 61. & \begin{tabular}{|c|}
$\begin{array}{c}\text { Tetrastigma obtectum } \\
\text { (Wall. ex Law.) Planch. } \\
\text { ex Franch. }\end{array}$ \\
\end{tabular} & Uttar Pradesh, Sikkim, Arunachal Pradesh and Manipur & Nepal, Bhutan and China (Yunnan). \\
\hline 62. & $\begin{array}{l}\text { Tetrastigma planicule } \\
\text { (Hook. f.) Gag. }\end{array}$ & Sikkim, Arunachal Pradesh Assam and Meghalaya & Laos, Sri Lanka, Vietnam \\
\hline 63. & $\begin{array}{l}\text { Tetrastigma } \\
\text { rumicispermum } \\
\text { (Lawson) Planch. }\end{array}$ & $\begin{array}{c}\text { Assam, Sikkim, West Bengal, Arunachal Pradesh and } \\
\text { Meghalaya }\end{array}$ & $\begin{array}{c}\text { Nepal, Bhutan, Myanmar, Vietnam, Laos and } \\
\text { Malaysia. }\end{array}$ \\
\hline 64. & \begin{tabular}{|c|} 
Tetrastigma serrulatum \\
(Roxb.) Planch.
\end{tabular} & $\begin{array}{l}\text { Himachal Pradesh, Uttar Pradesh, Assam, Sikkim, } \\
\text { Arunachal Pradesh and Meghalaya }\end{array}$ & \begin{tabular}{|c}
$\begin{array}{c}\text { Nepal, Bhutan, Bangladesh, China (Yur } \\
\text { and Thailand. }\end{array}$ \\
\end{tabular} \\
\hline 65. & $\begin{array}{l}\text { Tetrastigma sulcatum } \\
\text { (Lawson) Gam. }\end{array}$ & Goa, Andhra Pradesh, Kerala, Karnataka and Tamil Nadu & 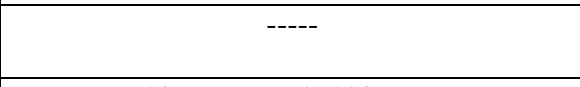 \\
\hline 6. & \begin{tabular}{|c|} 
Vitis flexuosa var. \\
parvifolia (Roxb.) Gag.
\end{tabular} & $\begin{array}{l}\text { Kashmir, Himachal Pradesh, Uttar Pradesh, West Bengal, } \\
\text { Assam and Manipur. }\end{array}$ & Pakistan, Nepal, China. \\
\hline 87. & $\begin{array}{l}\text { Vitis heyneana Roemer } \\
\text { and Schult. }\end{array}$ & $\begin{array}{c}\text { Himachal Pradesh West Bengal, Assam, Sikkim, Andhra } \\
\text { Pradesh, and Meghalaya }\end{array}$ & Nepal, Bhutan, West China and Myanmar. \\
\hline 68. & Vitis labrusca L. & Cultivated in Certain Parts of India. & Pakistan and Nepal. \\
\hline 69. & $\begin{array}{l}\text { Vitis jacquemontii R. } \\
\text { Parker }\end{array}$ & Himachal Pradesh and Uttar Pradesh. & Native of Europe. \\
\hline 70. & Vitis vinifera L. & Cultivated in Certain Parts of India. & Native of W. Asia. \\
\hline 71. & Leea aequata $\mathrm{L}$. & $\begin{array}{c}\text { Uttar Pradesh, Bihar, West Bengal, Sikkim, Assam, } \\
\text { Arunachal Pradesh, Odisha, Madhya Pradesh, Maharashtra, } \\
\text { Karnataka, Tamil Nadu, and Andaman islands. } \\
\end{array}$ & $\begin{array}{l}\text { Nepal, Bhutan, Bangladesh, Myanmar, } \\
\text { Vietnam, Cambodia, Thailand, Malaysia, } \\
\text { Singapore, Laos, Indonesia and Philippines. }\end{array}$ \\
\hline 72. & Leea alata Edgew. & $\begin{array}{c}\text { Gangetic plains, eastern and central India, ascending upto } \\
1500 \text { m in the Himalaya. Himachal Pradesh, Uttar Pradesh, } \\
\text { Bihar, West Bengal, Sikkim, Assam, Arunachal Pradesh, } \\
\text { Meghalaya, Odisha and Madhya Pradesh. }\end{array}$ & Nepal and Bhutan. \\
\hline 73. & $\begin{array}{l}\text { Leea angulata Korth. } \\
\text { Ex Miq. }\end{array}$ & Coastal belts, Nicobar Islands. & $\begin{array}{l}\text { Peninsular Thailand, Malaysia, Singapore, } \\
\text { Indonesia and Philippines. }\end{array}$ \\
\hline 74. & $\begin{array}{l}\text { Leea compactiflora } \\
\text { Kurz. }\end{array}$ & $\begin{array}{c}\text { Evergreen forest up to 2000m, Uttar Pradesh (Terai), West } \\
\text { Bengal, Sikkim, Arunachal Pradesh, Nagaland, Assam, } \\
\text { Manipur, Mizoram and Meghalaya. }\end{array}$ & $\begin{array}{l}\text { Bhutan, Bangladesh, Myanmar, Laos, North } \\
\text { Vietnam, and China. }\end{array}$ \\
\hline 75. & Leea grandifolia Kurz. & Coastal Areas, Andaman and Nicobar Islands. & ----- \\
\hline 76. & $\begin{array}{l}\text { Leea asiatica }(\mathrm{L} .) \\
\text { Ridsdale }\end{array}$ & $\begin{array}{l}\text { Evergreen, deciduous and lower mountain forests, up to } \\
\text { 2250m in the Himalaya, Uttar Pradesh, grasslands in the } \\
\text { plains, Jammu and Kashmir, Himachal Pradesh, Madhya } \\
\text { Pradesh, Bihar, West Bengal, Sikkim, Tamil Nadu, Kerala, } \\
\text { Arunachal Pradesh, Assam, Odisha, Nagaland, Manipur, } \\
\text { Mizoram, Andhra Pradesh, Meghalaya, Rajasthan, Bihar, } \\
\text { Deccan (Sandur hills) Maharashtra, Karnataka, and } \\
\text { Andaman Islands. }\end{array}$ & $\begin{array}{l}\text { Nepal, Laos, Bhutan, Madagascar, Myanmar, } \\
\text { Bangladesh, Vietnam, Thailand and China. }\end{array}$ \\
\hline 77. & $\begin{array}{l}\text { Leea guineensis } \mathrm{G} . \\
\text { Don. }\end{array}$ & $\begin{array}{l}\text { Uttar Pradesh, Sikkim, Assam, Manipur, Tamil Nadu, } \\
\text { Meghalaya, Maharashtra and Andaman Islands. }\end{array}$ & \begin{tabular}{|c|} 
Myanmar, Thailand, Laos, Taiwan, Bourbon \\
Malaysia, Indonesia, New Guinea, Central \\
West and East Africa, Micronesia, Mauritius. \\
Philippines and Tropical Madagascar. \\
\end{tabular} \\
\hline
\end{tabular}


International Journal of Science and Research (IJSR)

ISSN (Online): 2319-7064

Index Copernicus Value (2013): 6.14 | Impact Factor (2014): 5.611

\begin{tabular}{|c|c|c|c|}
\hline 78. & $\begin{array}{l}\text { Leea indica (Burm.f.) } \\
\text { Merr. }\end{array}$ & $\begin{array}{l}\text { Punjab, Uttar Pradesh, Bihar, West Bengal, Sikkim, } \\
\text { Arunachal Pradesh, Assam, Goa, Nagaland, Mizoram, } \\
\text { Tripura, Madhya Pradesh, Meghalaya, Odisha, } \\
\text { Maharashtra, Andhra Pradesh, Tamil Nadu, Kerala and } \\
\text { Andaman \& Nicobar Islands. }\end{array}$ & \begin{tabular}{|c|} 
Sri Lanka, Nepal, Bangladesh, Fiji, Myanmar, \\
Singapore, Indonesia, New Guinea, \\
Philippines, Laos, Vietnam, Cambodia, \\
Thailand, China, Malaysia, N. Australia, \\
Solomon Island and Santa Cruz Island. \\
\end{tabular} \\
\hline 79. & $\begin{array}{l}\text { Leea macrophylla } \\
\text { Roxb. ex Hornem. }\end{array}$ & $\begin{array}{l}\text { Sub Himalayan tract upto 2250m and Western Ghats, Uttar } \\
\text { Pradesh, Bihar, West Bengal, Sikkim, Assam, Meghalaya, } \\
\text { Odisha, Madhya Pradesh, Maharashtra, Andhra Pradesh, } \\
\text { Karnataka, Tamil Nadu, Kerala and Andaman island. }\end{array}$ & $\begin{array}{l}\text { Nepal, Bhutan, Bangladesh, up to 2250m } \\
\text { Myanmar, Laos, Cambodia and Thailand. }\end{array}$ \\
\hline 80. & Leea setuligera Clarke & Assam, Maharashtra (Khandala) and Karnataka (Concan). & Thailand and China . \\
\hline 81. & $\begin{array}{l}\text { Leea rubra Blume ex } \\
\text { Spreng. }\end{array}$ & West Bengal, Assam and Meghalaya. & $\begin{array}{c}\text { Bangladesh, Myanmar, Thailand, Vietnam, } \\
\text { Cambodia, Malaysia, Philippines, Laos, New } \\
\text { Guinea and North Australia }\end{array}$ \\
\hline 82. & Yua thomsonii Li & Assam & Nepal, China. \\
\hline
\end{tabular}

\section{Acknowledgement}

Author is thankful to Prof. R.P. Upadhyay, PVC, Nalanda Open University, Prof. A.K Pandey, Delhi University for all the support during the completion of work.

\section{References}

[1] Angiosperm Phylogeny Group (2009). An update of the Angiosperm Phylogeny Group Classification for the order and families of flowering plants APG III. Bot. J. Linn. Soc. 161: 105 - 121.

[2] Chen, I., and Manchester, S. R. (2007). Seed morphology of modern and fossil Ampelocissus (Vitaceae) and implications for phytogeography. Amer. J. Bot. 94: 1534-1553.

[3] Donoghue, M. J.; Bell, C. D. and Li, J. H. (2001). Phylogenetic patterns in northern hemisphere plant geography. Int. J. Plant Sci. 162: 541-552.

[4] Jain, S.K. and R.R. Rao (1977). A Handbook of Field and Herbarium Methods. Today and Tomorrow's Printers and Publishers, New Delhi.

[5] Li, C. L. (1990). Yua C.L. Li - A new genus of Vitaceae. Act. Bot. Yunn. 12: $1-10$.

[6] Manchester, S. R. (1994). Fruits and seeds of the middle Eocene Nut Beds fl ora, Clarno Formation, Oregon. Palaeontographica Americana 58:1-205.

[7] Naithani, B.D. (2000). Leeaceae. Singh, N.P., Vohra, J.N., Hajra, P.K. \& Singh, D.K. (eds.), Flora of India, Calcutta: Botanical Survey of India. Vol. 5: 325-342.

[8] Nie, Z.L.; Sun, H.; Chen, Z.D.; Meng, Y.; Manchester, S.R. \& Wen, J. (2010). Molecular phylogeny and biogeographic diversification of Parthenocissus (Vitaceae) disjunct between Asia and N. America. Amer. J. Bot. 97: 1342-1353.

[9] Peros, J. P.; Berger, G.; Portemont, A.; Boursiquot, J.M. and Lacombe, T. (2011). Genetic variation and biogeographic diversification of Parthenocissus (Vitaceae) J. Biogeo. 38: 471-486.

[10] Punt, W.; Marks, A. and Hoen, P.P. (2003). The northwest European pollen flora, 64 Vitaceae. Rev. Palaeobot. Palynol., 123, 67-70.

[11] Shetty, B.V. and P. Singh (2000). Vitaceae.In: Singh, N.P., Vohra, J.N., Hajra, P.K. \& Singh, D.K. (eds.), Flora of India, Calcutta. Botanical Survey of India 5: 246-324.

[12] Soejima, A. and J. Wen (2006). Phylogenetic analysis of the grape family (Vitaceae) based on three chloroplast markers. Amer. J.Bot. 93: 278 287.

[13] Wen, J. (1999). Evolution of eastern Asian and eastern North American disjunct distributions in flowering plants. Ann. Rev. Eco. System. 30: 421-455.

[14] Wen, J. 2007a. Leeaceae. The Families and Genera of Vascular Plants, Vol. 9 (ed. by K. Kubitzki), pp. 220224. Springer-Verlag, Berlin Heidelberg, Germany.

[15] Xiang, Q-Y.; Soltis, D.E. (2001). Dispersal-vicariance analyses of intercontinental disjunctions: historical biogeographical implications for angiosperms in the northern hemisphere. Int. J. Plant Science 162, S29S39.

[16] Xiang, Q.-P.; Soltis, D.E. \& Soltis, P.S. (1998). The eastern Asian and eastern and western North American floristic disjunction: congruent phylogenetic patterns in seven diverse genera. Mol. Phyl. Evol. 10: 178-190. 